Check for updates

Cite this: RSC Adv., 2019, 9, 7147

Received 7th December 2018

Accepted 17th February 2019

DOI: $10.1039 / c 8 \mathrm{ra10071c}$

rsc.li/rsc-advances

\section{Structure and properties of halogen-free flame retardant and phosphorus-containing aromatic poly(1,3,4-oxadiazole)s fiber $\dagger$}

\author{
Pengqing Liu, (D) *a Linan Dong, ${ }^{\mathrm{b}}$ Lei $\mathrm{Wu}{ }^{\mathrm{a}}$ Luxian Zeng ${ }^{\mathrm{a}}$ and Jianjun $\mathrm{Xu}^{\mathrm{a}}$
}

In order to improve the flame retardance of aromatic polyoxadiazole ( $p$-POD) fiber, a series of phosphoruscontaining PODs (pho-POD) were synthesized by introducing triaryl phosphine oxide (TPO) units into the main chains of $p$-POD using hydrazine sulfate, terephthalic acid and bis(p-carboxy)phenyl phosphine oxide (BCPPO) as monomers, and then halogen-free flame resistant pho-POD fibers were obtained from wet spinning. The structure and properties of the pho-POD fibers were characterized and measured in detail using the methods of wide-angle X-ray diffraction (WAXD), scanning electron microscopy (SEM), thermogravimetric analysis (TGA), the limiting oxygen index (LOI), oxygen bomb calorimeter, Pyrolysis-Gas Chromatography/Mass Spectrometry (Py-GC/MS) etc. The results show that the introduction of TPO units resulted in the weakening of the crystallization ability, the formation of the poriferous and lax interior structure, the slight decrease in the thermal stability and mechanical properties of the POD fibers. However, the value of LOI obviously increased from $28 \%$ to $35 \%$, and the gross heat of combustion (GHC) decreased from $19.72 \mathrm{MJ} \mathrm{kg}^{-1}$ to $17.84 \mathrm{MJ} \mathrm{kg}^{-1}$ with the increase in the content of the BCPPO. Moreover, the combustion residue of pho-POD fiber revealed a smooth, dense and non-porous carbon layer, which could effectively play a role of oxygen barrier and enhance the flame resistance. From the above results, it can be concluded that the flame resistance of the POD fiber could be improved significantly after introducing the TPO unit. The results of Py-GC/MS illustrate that the TPO unit of pho-POD could inhibit the production of volatile products, which could be confirmed that the mechanism of enhancing the flame retardancy by introducing TPO units was mainly the flame retardation of the condensed phase.

\section{Introduction}

Aromatic poly(1,3,4-oxadiazole)s ( $p$-POD) fibers is a new type of high performance fiber with an excellent thermal stability, well electrical insulation, good chemical stability, special photoluminescence and electric properties. Therefore, the $p$-POD fibers have very good application prospects in the heat-resistant filter materials, electrical insulating material, protective products, industrial fabric, etc. However, $p$-POD fiber has some obvious disadvantages, particularly poor ultraviolet (UV) light resistance and flame retardancy, which greatly limit its development and the scope of its applications. In the past few years, considerable efforts were made to study its UV light resistance, including the effect of UV irradiation and the mechanisms of photodegradation, ${ }^{\mathbf{1 , 2}}$ the methods to improve its UV resistance, ${ }^{3-9}$ the function of oxygen during the photodegradation process $^{\mathbf{1 0}}$ and so on. However, the disadvantage of the poor

${ }^{a}$ College of Polymer Science \& Engineering, Sichuan University, Chengdu 610065, China. E-mail: liupq@scu.edu.cn; Tel: +86-28-8546-2013

${ }^{b}$ Chongqing Academy of Metrology and Quality Inspection, Chongqing 401120, China $\dagger$ Electronic supplementary information (ESI) available. See DOI: 10.1039/c8ra10071c flame resistance of the POD fibers did not attract much attention. Zhang et al. evaluated the fire retardant performance of the POD fibers using the methods of cone calorimetry, Fourier transform infrared spectroscopy (FT-IR) and pyrolysis-gas chromatography/mass spectrometry (Py-GC/MS). ${ }^{\mathbf{1 1}}$ Pankina et al. synthesized a bromine-containing POD using brominecontaining terephthalic acids and prepared the fibers based on this polymer. They examined the possibility of using bromine-containing terephthalic acids as monomers for the preparation of fire-resistance fibers. ${ }^{12}$

Bis(4-carboxyphenyl)phenyl phosphine oxide (BCPPO) is a highly efficient flame retardant containing triaryl phosphine oxide (TPO) and dicarboxy functional groups, which has been used as a third monomer to prepare many inherently flameretardant polymers, such as poly(trimethylene terephthalate), ${ }^{13}$ poly(ethylene terephthalate), ${ }^{14,15}$ polyarylate, ${ }^{\mathbf{1 6}}$ polyamide $66,{ }^{17}$ soluble aromatic copolyamides ${ }^{18}$ etc. From these literatures, it can be found that the copolymers exhibited improved flame retardance, hydrolytic stability and thermal stability, when BCPPO was incorporated into their main chains.

In the present study, in order to improve the flame retardance of the POD fiber, a series of phosphorus-containing PODs (pho-PODs) were prepared through a one-step method with 
three stages, including prepolymerization, polycondensation and cyclodehydration, where Hydrazine sulfate (HS), Terephthalic acid (TPA) and BCPPO were copolymerized in different proportions, and then the POD fibers were spun by wet spinning directly using the solution obtained by polymerization. The focus of this study was on the preparation of the halogen-free flame retardant POD fibers, analyzing the molecular structures of the POD fibers and theirs flame resistant behavior, and understanding the flame retarding mechanism. The wide-angle X-ray diffraction (XRD), scanning electron microscopy (SEM), limiting oxygen index (LOI), thermogravimetric analysis (TGA), oxygen bomb calorimeter and Py-GC/MS were used to characterize the aggregate and morphological structures, flame resistance, thermal stability, thermal decomposition and flame resistance mechanisms.

\section{Experimental}

\section{Materials}

Dichlorophenylphosphine (DCPP, ACROS), terephthalic acid (TPA, ACROS), oleum (industrial grade), ethanol, potassium permanganate and 98\% sulfuric acid were used as received. Sublimed sulfur and anhydrous aluminum chloride were used after vacuum drying. Hydrazine sulfate (HS) was recrystallized before use. Toluene and pyridine were dehydrated using $3 \mathrm{~A}$ molecular sieve. All other materials were analytically pure unless otherwise stated and were purchased from Kelong Chemical Reagent Factory (Chengdu, China) except DCPP and TPA.

\section{Synthesis of bis(4-carboxyphenyl)phenyl phosphine oxide (BCPPO)}

The synthetic route of BCPPO is shown in Scheme 1. Dichlorophenylphosphine sulfide (DCPPS) was prepared by the FriedelCrafts reaction of DCPP and sublimed sulfur with anhydrous aluminum chloride as catalyst in the first step. The obtained DCPPS reacted with excess toluene to obtain the crude product of bis(4-methylphenyl)phenylphosphine sulfide (BMPPS) in the second step. BMPPS was oxidized twice to BCPPO using potassium permanganate as an oxidizing agent in the third step. A typical procedure is given as $62.0 \mathrm{~g}$ of anhydrous aluminums chloride and $69.8 \mathrm{~g}(0.39 \mathrm{~mol})$ of DCPP were added in a $500 \mathrm{~mL}$ three-necked flask equipped with a magnetic stirrer, a reflux condenser and an acid gas receiver. The mixture was slightly heated to $60{ }^{\circ} \mathrm{C}$ under gentle stirring to form a homogeneous solution. $12.8 \mathrm{~g}(0.4 \mathrm{~mol})$ sublimed sulfur powder was added in batches slowly, and then heated to $80-84{ }^{\circ} \mathrm{C}$ and stirred for $4 \mathrm{~h}$. $205 \mathrm{~mL}$ of toluene was directly added to the above three-necked flask, then the reaction mixture was heated to $110-125{ }^{\circ} \mathrm{C}$ and maintained for $8 \mathrm{~h}$, and then the excess toluene was distilled under a vacuum distillation method. After cooling to room temperature, the reaction mixture was poured into ice water slowly under vigorous stirring. The crude product was washed repeatedly with deionized water and refluxed-ethanol, respectively, and the white powder of BMPPS was obtained after filtration and drying. $30.6 \mathrm{~g}$ ( $0.1 \mathrm{~mol})$ of BMPPS, $120 \mathrm{~mL}$ of pyridine and $120 \mathrm{~mL}$ of water were added to a $500 \mathrm{~mL}$ three-necked flask, and the mixture was heated to $90{ }^{\circ} \mathrm{C}$ with stirring. $95 \mathrm{~g}(0.6 \mathrm{~mol})$ of $\mathrm{KMnO}_{4}$ was added in portions over $3 \mathrm{~h}$ and reacted at $95{ }^{\circ} \mathrm{C}$ for $8 \mathrm{~h}$. After cooling to room temperature, the mixture was filtered, and the filtrate was acidified to $\mathrm{pH} 2$ with dilute hydrochloric acid. The white solid product was obtained after filtration. In another $500 \mathrm{~mL}$ three-necked flask, the obtained solid product was dissolved in an appropriate amount of $\mathrm{NaOH}$ aqueous solution, and the $\mathrm{pH}$ was adjusted at 10-12, and then $30 \mathrm{~g}(0.2$ mol) of $\mathrm{KMnO}_{4}$ was added and the temperature was raised to 90$100{ }^{\circ} \mathrm{C}$ for $8 \mathrm{~h}$. After cooling, the mixture was acidified with hydrochloric acid to obtain a white solid, which was filtered and washed with distilled water several times, and dried to give a crude product. The crude BCPPO was washed repeatedly with boiling ethanol to obtain the pure product with the total yield of $70 \%$. The structure of BCPPO was confirmed by its melting point, FT-IR and ${ }^{1} \mathrm{H}$ NMR spectra (shown in Fig. S1-S3 in ESI $\dagger$ ): m.p. $343{ }^{\circ} \mathrm{C}$ (determined by DSC at a heating rate of $5{ }^{\circ} \mathrm{C} \mathrm{min}^{-1}$ under nitrogen atmosphere); FT-IR (KBr): $3300-2500 \mathrm{~cm}^{-1}\left(-\mathrm{COOH}, \nu_{\mathrm{O}-}\right.$ H), $1722 \mathrm{~cm}^{-1}\left(-\mathrm{COOH}, \nu_{\mathrm{C}=\mathrm{O}}\right), 1560-1400 \mathrm{~cm}^{-1}\left(-\mathrm{Ar}, \nu_{\mathrm{C}=\mathrm{C}}\right)$, $1146 \mathrm{~cm}^{-1}\left(-\mathrm{PO}, \nu_{\mathrm{P}=\mathrm{O}}\right) ;{ }^{1} \mathrm{H}$ NMR $\left(600 \mathrm{MHz}, \mathrm{DMSO}-d_{6}, \delta, \mathrm{ppm}\right)$ : $13.352\left(\mathrm{~s}, 2 \mathrm{H}, \mathrm{H}_{1}\right), 8.095\left(\mathrm{~d}, 4 \mathrm{H}, \mathrm{H}_{\mathrm{d}}\right), 7.776\left(\mathrm{~d}, 4 \mathrm{H}, \mathrm{H}_{3}\right), 7.670(\mathrm{~m}$, $3 \mathrm{H}, \mathrm{H}_{5}+\mathrm{H}_{6}$ ), 7.587 (d, 2H, $\mathrm{H}_{4}$ ).

\section{Preparation of $p$-POD and phosphorus-containing POD (pho- POD) solutions}

According to the synthetic route reported by the previous studies, ${ }^{19,20} p$-POD and a series of pho-PODs were prepared by a one-step method with three stages including

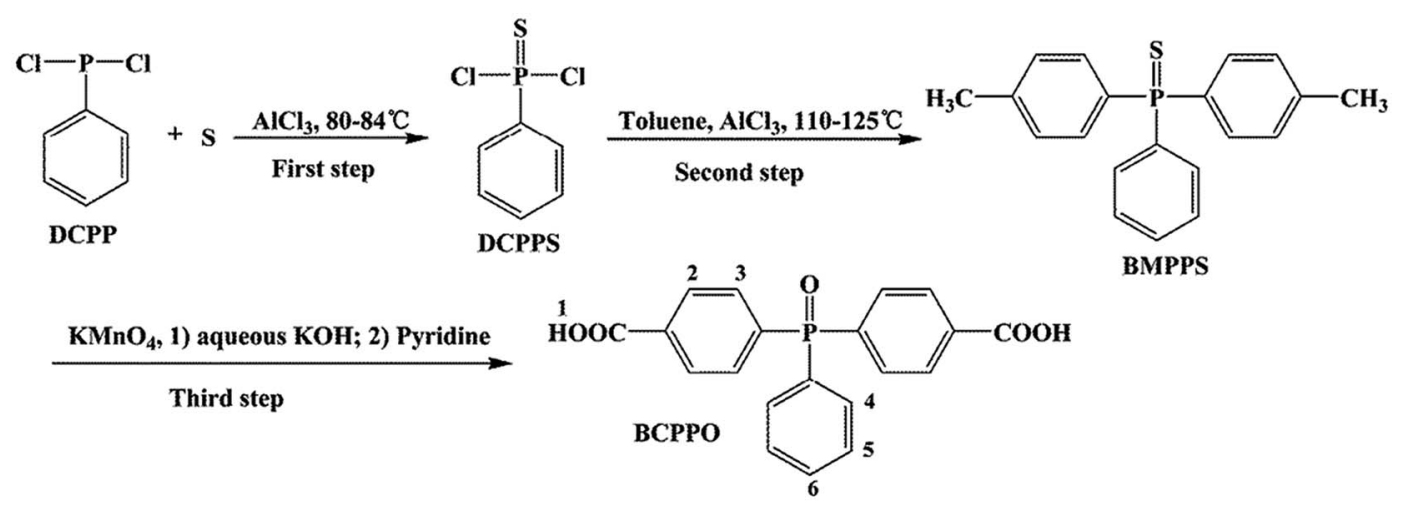

Scheme 1 Synthesis route of the monomer BCPPO. 
prepolymerization, polycondensation and cyclodehydration, as is shown in Scheme 2. HS, TPA and BCPPO were used as monomers, and $20 \%$ oleum was used as solvent and condensing agent. The detailed procedure can be described as follows: a $500 \mathrm{~mL}$ three-necked flask equipped with a mechanical stirrer, dry nitrogen inlet and condenser connected to a silica-gel desiccator was immersed in a thermostatic oil bath with precision of $1{ }^{\circ} \mathrm{C}$. Oleum and HS were added to the flask and stirred under nitrogen at $85{ }^{\circ} \mathrm{C}$. After HS being totally dissolved in oleum, BCPPO and TPA were added to the flask. The precondensation reaction was conducted at $85{ }^{\circ} \mathrm{C}$ for $3 \mathrm{~h}$, then continued to polymerize at $120^{\circ} \mathrm{C}$ for $2 \mathrm{~h}$. Subsequently, the reaction temperature was raised to $140{ }^{\circ} \mathrm{C}$, and the reaction was terminated by cyclization for about $2 \mathrm{~h}$ to obtain a pho-POD polymer solution. The obtained pho-POD solution was symbolized as pho-POD- $Y$, where $Y$ represented the molar ratio of BCPPO to the total molar of dicarboxylic acid. The codes, feed ratios and inherent viscosities of these PODs are summarized in Table 1.

\section{Wet-spinning of the $\boldsymbol{p}$-POD and pho-POD solutions}

The POD fibers were fabricated from the above obtained polymer solutions through the wet-spinning method. As is shown in Fig. 1, the processes of wet-spinning included degassing, metering, filtrating, extruding, coagulating, washing, drawing, take-up etc., which are described in details in our previous study. ${ }^{19,20}$

\section{Measurement and characterization}

FT-IR spectra were recorded on a Nicolet 560 spectrometer with a resolution of $1 \mathrm{~cm}^{-1}$ and a wavenumber range of $500-$
$4000 \mathrm{~cm}^{-1} \cdot{ }^{1} \mathrm{H}$ nuclear magnetic resonance $\left({ }^{1} \mathrm{H} \mathrm{NMR}\right)$ spectrum was measured using a Bruker $600 \mathrm{MHz}$ Ultra Shield NMR spectrometer. The chemical shifts were calibrated by using tetramethylsilane (TMS) as an internal standard, and the peak multiplicity was described as follows: s, singlet; d, doublet; $t$, triplet; $\mathrm{m}$, multiplet. The inherent viscosities of the pho-PODs were determined using an Ubbelohde capillary viscometer (4 - Ø0.9-1.0 mm, which meant the capillary diameter was 0.9$1.0 \mathrm{~mm}$ and the capacity of the measuring bulb was $4 \mathrm{~mL}$ ) at a concentration of $0.25 \mathrm{~g} \mathrm{dL}^{-1}$ in $98 \%$ concentrated sulfuric acid at $30 \pm 0.1{ }^{\circ} \mathrm{C}$. The wide angle X-ray diffraction (WAXD) patterns were measured at room temperature with a $\mathrm{X}^{\prime}$ Pert Pro X-ray diffractometer at the scanning rate of $3.5^{\circ} \mathrm{min}^{-1}$ over a range of scattering angle from $5^{\circ}$ to $45^{\circ}$. Scanning electron micrographs (SEM) were recorded using a JSM-5900LV scanning electron microscope with an accelerating voltage of $40 \mathrm{kV}$. The mechanical properties of the pho-POD fibers were measured using an YG001A-1 single fiber electric strength tester with a $0.1 \mathrm{~N}$ load cell. The tests were conducted on single fiber with a crosshead speed of $20 \mathrm{~mm} \mathrm{~min} \mathrm{~m}^{-1}$ and a gauge length of 20 $\mathrm{mm}$. Thermal stabilities were conducted using a TA SDT Q600 thermal analyzer at a heating rate of $10^{\circ} \mathrm{C} \mathrm{min}^{-1}$ from $180^{\circ} \mathrm{C}$ to $800{ }^{\circ} \mathrm{C}$ in flowing nitrogen $\left(100 \mathrm{~cm}^{3} \mathrm{~min}^{-1}\right)$.

Limiting oxygen index (LOI) was performed on an ATLAS LOI-1045GO5 apparatus. The specimens were pho-POD braids with a dimension of $3 \mathrm{~mm}$ in thickness, $6 \mathrm{~mm}$ in width and $100 \mathrm{~mm}$ in length. A specimen was positioned vertically in a transparent test column and a mixture of oxygen and nitrogen was forced upward through the column. The specimen was ignited at the top, and the oxygen concentration was adjusted
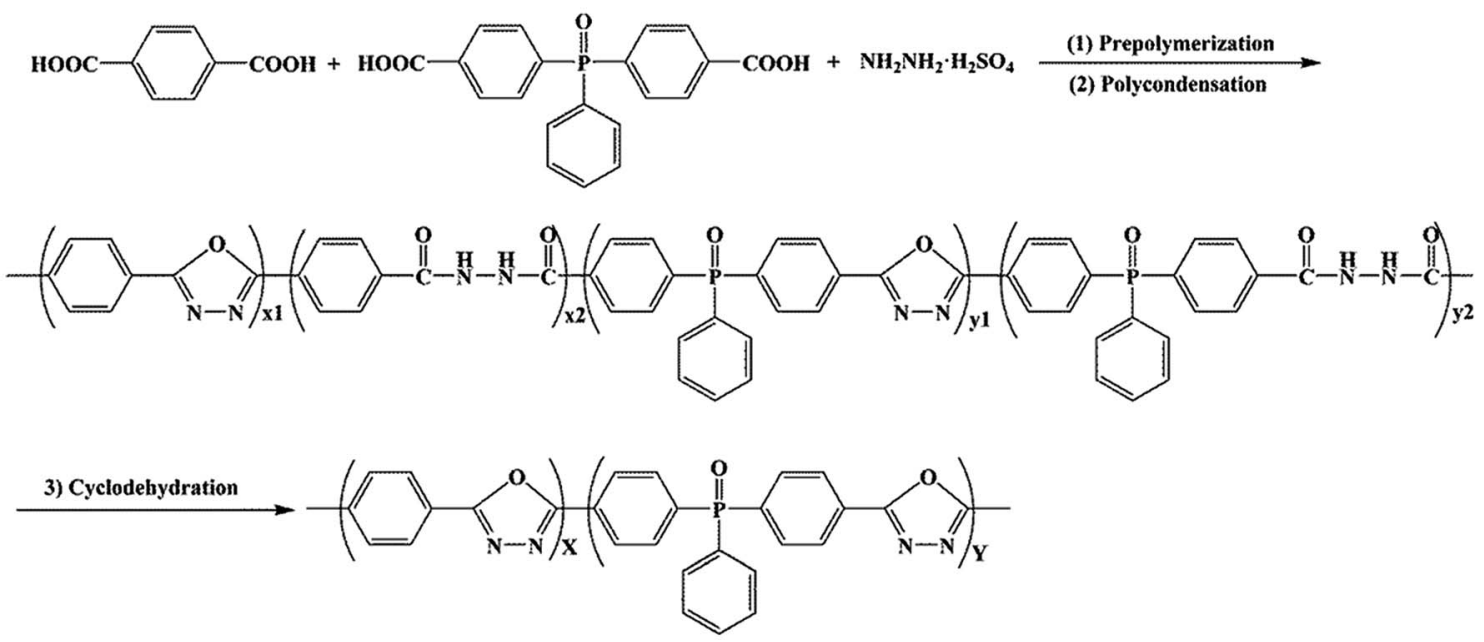

Scheme 2 Synthetic route of the pho-PODs.

Table 1 Feed molar ratios and inherent viscosities of the as-prepared PODs

\begin{tabular}{lllll}
\hline Polymer code & BCPPO $:$ TPA $:$ HS & $\eta_{\text {inh }}\left(\mathrm{dL} \mathrm{g}^{-1}\right)$ & Polymer code & BCPPO : TPA : HS \\
\hline$p$-POD & $0: 100: 105$ & 2.83 & pho-POD-12.5 & $12.5: 87.5: 105$ \\
pho-POD-5 & $5: 95: 105$ & 2.11 & pho-POD-15 & $15: 85: 105$ \\
pho-POD-7.5 & $7.5: 92.5: 105$ & 1.98 & pho-POD-20 & $20: 80: 105$ \\
pho-POD-10 & $10: 90: 105$ & 1.95 & - & -
\end{tabular}




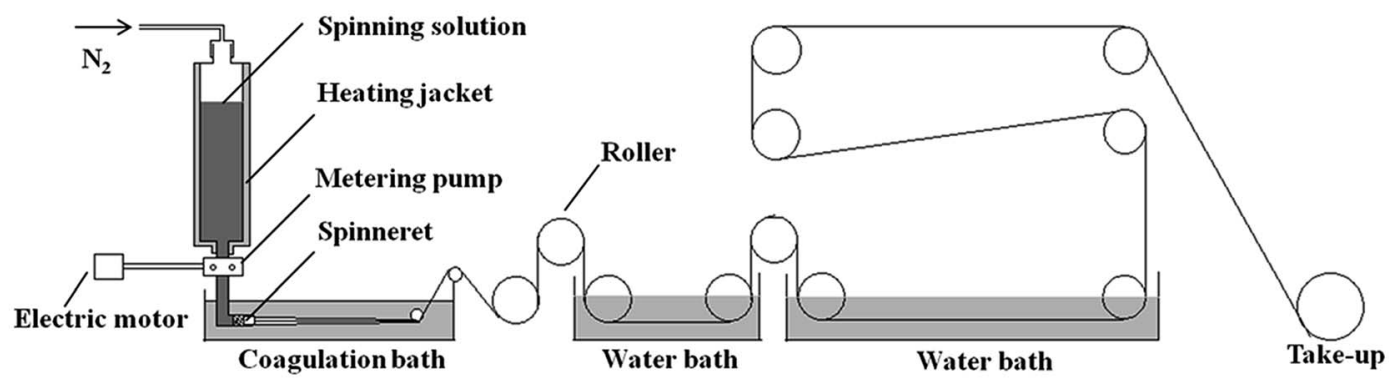

Fig. 1 Schematic of the wet-spinning method.

until the specimen just supports combustion. The gross heat of combustion (GHC) of the specimen was measured by an oxygen bomb calorimeter (Model C5000, Germany) according to a standard procedure, GB/T 14402-2007/ISO 1716:2002. The thermal degradations of the POD fibers were systematically studied using the combinations of Pyrolysis-Gas Chromatography/Mass Spectrometry (Py-GC/MS) and TG-FTIR methods. The Py-GC/MS was conducted using a PY-2020 is Single-Shot Pyrolyzer manufactured by Frontier Laboratories Ltd. and attached to a GCMS-QP2010 gas chromatography mass spectrometer manufactured by Shimadzu. The samples were

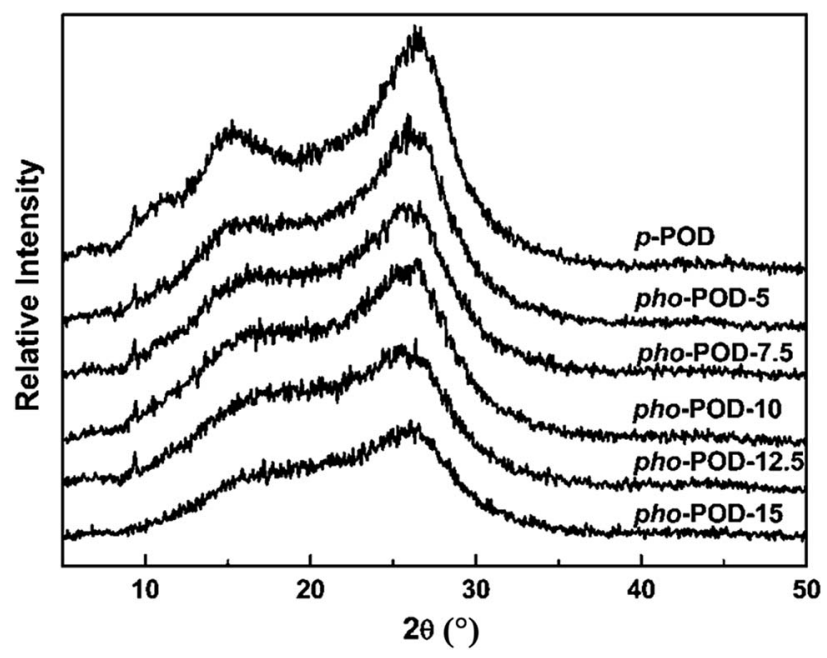

Fig. 2 WAXD patterns of the pho-POD fibers.
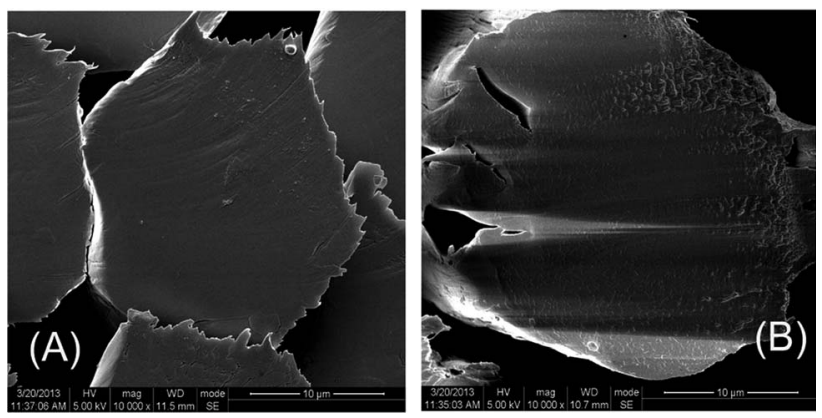

Fig. 3 Cross-section images of the $p$-POD (A) and pho-POD-15 (B) fibers.
Table 2 Mechanical properties of the pho-POD fibers

\begin{tabular}{llll}
\hline & \multicolumn{2}{l}{ Tensile strength } & \\
\cline { 2 - 3 } Sample code & (cN/dtex) & (MPa) & $\begin{array}{l}\text { Elongation at } \\
\text { break }(\%)\end{array}$ \\
\hline$p$-POD & 2.83 & 368 & 25.6 \\
pho-POD-5 & 2.60 & 338 & 24.8 \\
pho-POD-7.5 & 2.20 & 286 & 20.8 \\
pho-POD-10 & 2.10 & 273 & 22.8 \\
pho-POD-12.5 & 2.10 & 273 & 23.4 \\
pho-POD-15 & 1.83 & 238 & 21.3 \\
pho-POD-20 & 1.52 & 198 & 18.5
\end{tabular}

placed in a quartz sample tube, and pyrolysed at a temperature of $500{ }^{\circ} \mathrm{C}, 550{ }^{\circ} \mathrm{C}, 600{ }^{\circ} \mathrm{C}$ and $700{ }^{\circ} \mathrm{C}$, respectively. The pyrolysis products were separated on an Ultra-5MS (5\% of diphenylcompounds) column $(30 \mathrm{~m} \times 0.25 \mathrm{~mm} \times 0.25 \mu \mathrm{m})$ using helium carrier gas and identified by interpretation of their EI mass spectra and comparison to NIST 107. The GC was programmed from $40^{\circ} \mathrm{C}$ (held for $3 \mathrm{~min}$ ) to $280^{\circ} \mathrm{C}$ (held for $10 \mathrm{~min}$ ) at a heating rate of $10{ }^{\circ} \mathrm{C} \mathrm{min}^{-1}$. The MS was operated in EI mode $(70 \mathrm{eV})$ with a source temperature of $200{ }^{\circ} \mathrm{C}$. The GC/MS interface temperature was $280{ }^{\circ} \mathrm{C}$. TG-FTIR was performed using a Perkin Elmer Pyris-1 TG analyzer and Nicolet IR-560 infrared spectrometer equipped with a TG-FTIR interface. The sample was heated at the rate of $10{ }^{\circ} \mathrm{C} \mathrm{min}^{-1}$ from an ambient

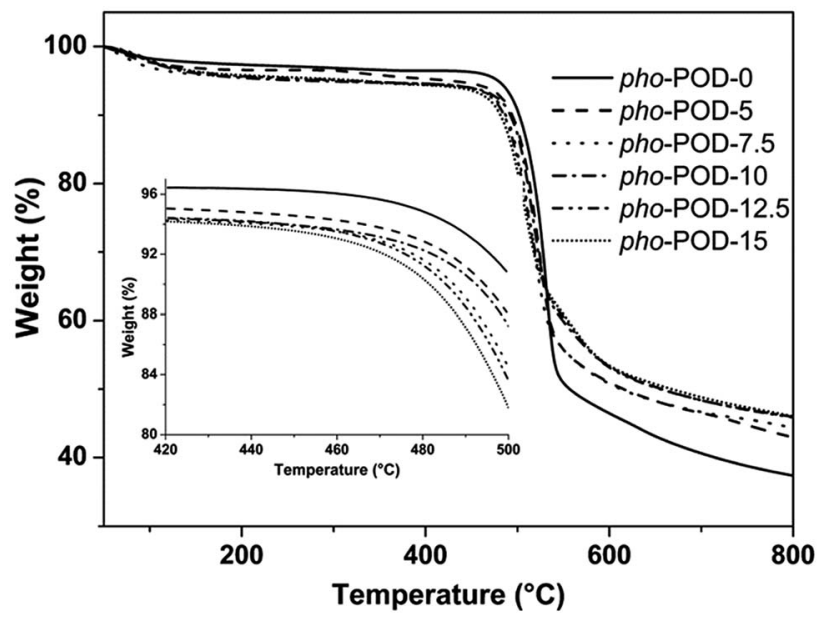

Fig. 4 TGA curves of the POD fibers. 


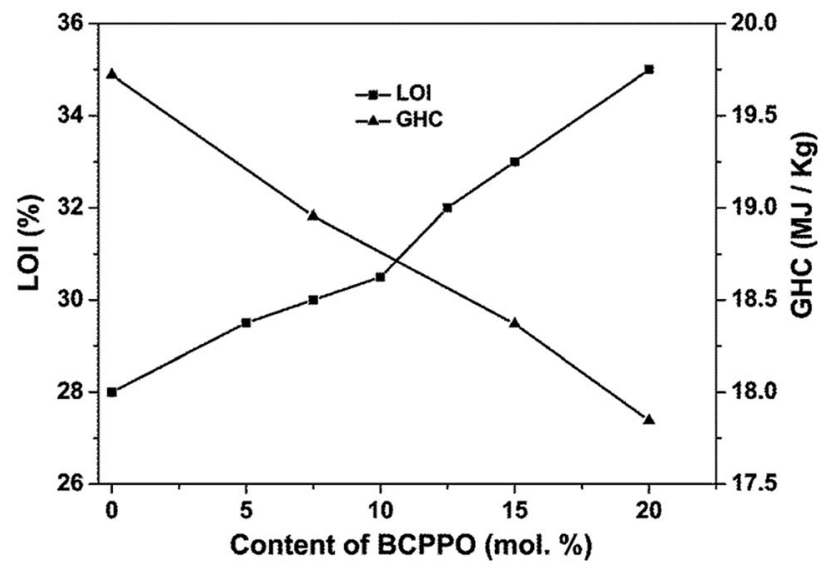

Fig. 5 LOI and GHC of pho-POD with different BCPPO content.

temperature to $800{ }^{\circ} \mathrm{C}$ under a steady flow of nitrogen $(100$ $\mathrm{mL} \min ^{-1}$ ) and the volatile gas produced from thermal decomposition was recorded online by FT-IR.

\section{Results and discussion}

\section{Crystallization behaviors of the fibers}

The crystallization behaviors of the POD fibers were studied by WAXD, and their WAXD patterns at room temperature are shown in Fig. 2. It can be found that $p$-POD fiber has two weak crystalline peaks at the diffraction angles $(2 \theta)$ of $14^{\circ}$ and $27^{\circ}$ in its WAXD pattern, suggesting $p$-POD belongs to semi-crystalline polymer; however, the crystallization ability is very poor due to its rigid and asymmetric molecular chains. After introducing TPO moiety, the diffraction peaks of pho-POD gradually weaken until become a dispersed peak as the content of TPO moiety increases, demonstrating pho-PODs belong to amorphous materials. It can be explained that the introduction of TPO destroys the chemical regularity of POD's molecular chains, which leads to the reduction in the crystalline ability.

\section{Morphology of POD fibers}

The morphological structure of the wet-spinning fiber is controlled by the diffusion of solvent from the polymer solution and the counter-diffusion of the non-solvent into the polymer solution during the coagulation process. As shown in Fig. 3, the cross-section morphologies of the POD fibers were observed using SEM. It can be found that both $p$-POD fiber and pho-POD15 fiber have approximatively round cross sections at the same coagulation conditions; however, the interior structure of $p$ POD fiber is compact and homogeneous, while it is poriferous and lax for pho-POD-15 fiber. The phase separation always accompanies a severe contraction of the polymer volume in coagulation, which leads to the formation of a porous gel network in the solid phase. In general, the micropores in the fibers can be fully filled by the moved macromolecular chains before completely solidified, resulting in a compact and homogeneous structure. However, the introduction of the rigid TPO units reduces the movement ability of the POD molecular chains, leading to no enough time to fill the micro pores.
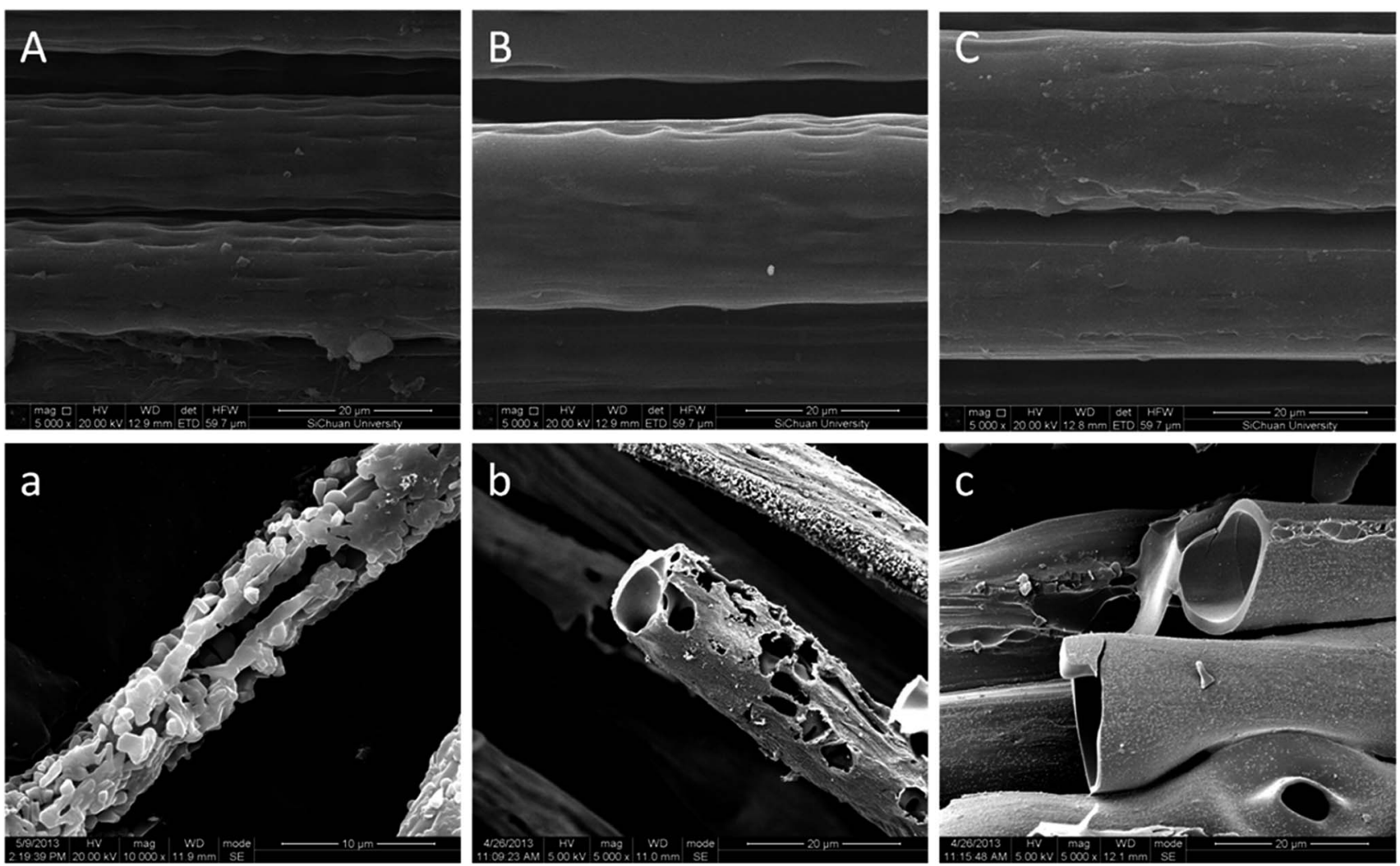

Fig. 6 SEM images of POD fibers before and after combustion: $p$-POD ( $A$ and a), pho-POD-7.5 (B and b), pho-POD-12.5 (C and c). 

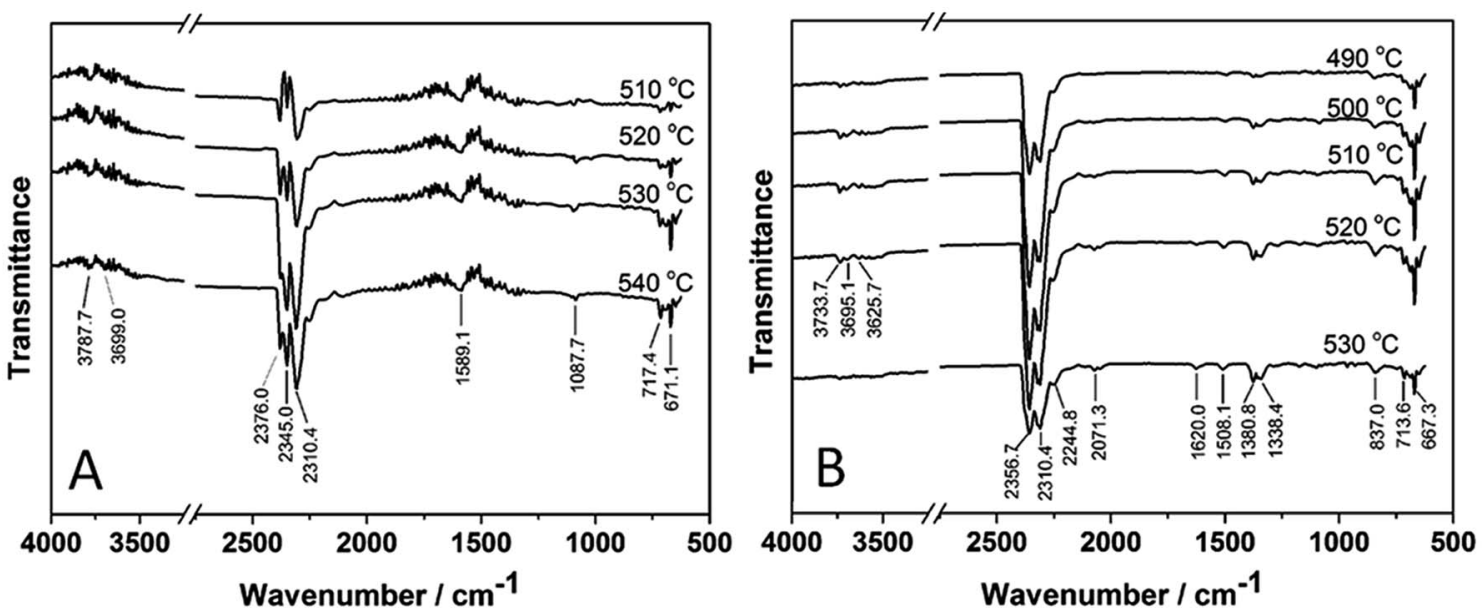

Fig. 7 FT-IR Spectra of the volatile gases released out during the thermal degradation of $p-P O D(A)$ and pho-POD-15 (B) fibers in nitrogen.

\section{Mechanical properties}

The mechanical properties of the fabricated POD fibers were measured by a single fiber electric strength tester, and the results are summarized in Table 2 . It can be seen that these POD fibers have the tensile strength of $198 \mathrm{MPa}-368 \mathrm{MPa}$ and the elongation at break of 18.5-25.6\%. However, both the tensile strength and the elongation at break of POD fibers decrease with the increase in the content of TPO units. To the best of our knowledge, the mechanical properties of the polymers depend on their relative molecular weights and aggregation structures. TPO unit has a bulky pendent phenyl, and its steric hindrance limits the reactivity of the carboxyl groups; therefore, the inherent viscosity of the POD, which represents the value of the relative molecular weight to some extent, decreases with the increase in the TPO content, as is summarized in Table 1. The decrease in the relative molecular weight and the crystallization ability, and the formation of the porous structure lead to the reduction in the mechanical properties of the POD fibers.

\section{Thermal stability}

The thermal stabilities of the fabricated POD fibers under nitrogen were studied by TGA, as shown in Fig. 4. The initial decomposition temperatures ( $T_{\mathrm{id}}, 5 \%$ weight loss) and the maximum decomposition temperatures $\left(T_{\mathrm{md}}\right.$, maximum rate of weight loss) of the as-prepared POD fibers are all above $480{ }^{\circ} \mathrm{C}$ and $500{ }^{\circ} \mathrm{C}$, respectively, while the char yields are above $37.0 \%$, which illustrate that these POD fibers have excellent thermal stabilities. As the content of TPO unit increases, the $T_{\mathrm{id}}$ and $T_{\mathrm{md}}$ of POD fiber decrease but the char yield increases (Table 3). It can be concluded that the introduction of TPO unit could slightly reduce the thermal stabilities of the POD fibers, which should be attributed to the comparative weak $\mathrm{P}-\mathrm{C}$ bond exited in the main chains of pho-PODs. However, the higher char yield of pho-PODs may be beneficial to improving the flame resistance.

\section{Flame resistance}

The flame and fire resistances of the POD fibers were characterized by the changes of LOI and GHC shown in Fig. 5. LOI is the minimum concentration of oxygen that will just support flaming combustion in a flowing mixture of oxygen and nitrogen, so it can be said that the higher the value of LOI is the lower the flammability of the material has. From Fig. 5, it can be found that the value of LOI obviously increases from $28 \%$ to $35 \%$ with the increase in the content of the BCPPO. The results show that the flame resistance of the POD fiber can be improved significantly after introducing the TPO unit. GHC is the energy released as heat when a unit mass of compound undergoes complete combustion with oxygen under standard conditions and the water in the combustion product condenses into liquid. The polymers best known for the heat and flame resistance include polytetrafluoroethylene (PTFE), polyimide (PI), poly $(m$ phenylene isophthalamide) (Nomex), poly $(p$-phenylene terephthalamide) (Kevlar), poly( $p$-phenylenebenzobisoxazole) (PBO), poly(phenylene sulfide) (PPS), poly(ether ether ketone) (PEEK)

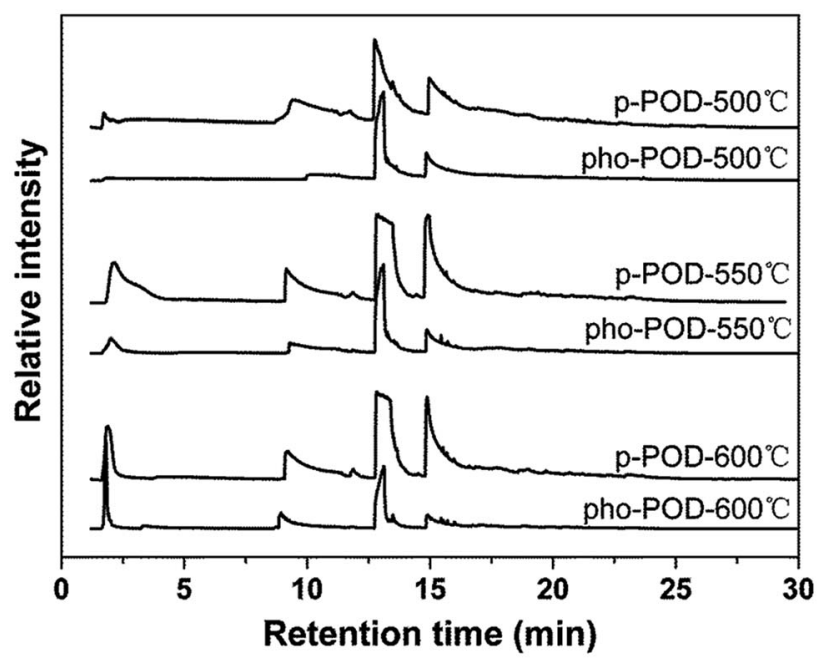

Fig. 8 Pyrolysates chromatograms of $p-P O D$ and pho-POD-15 fibers in helium at different temperatures. 
Table 3 Data analysis from TGA curves of the POD fibers

\begin{tabular}{llll}
\hline Sample code & $T_{\text {id }}\left({ }^{\circ} \mathrm{C}\right)$ & $T_{\text {md }}\left({ }^{\circ} \mathrm{C}\right)$ & $\begin{array}{l}\text { Char. yield } \\
(\%)\end{array}$ \\
\hline$p$-POD & 501 & 532 & 37.2 \\
pho-POD-5 & 494 & 525 & 42.7 \\
pho-POD-7.5 & 488 & 518 & 44.2 \\
pho-POD-10 & 493 & 515 & 45.8 \\
pho-POD-12.5 & 486 & 511 & 45.7 \\
pho-POD-15 & 482 & 508 & 45.8
\end{tabular}

etc., and their values of GHC are $6.68 \mathrm{MJ} \mathrm{kg}^{-1}, 26.03 \mathrm{MJ} \mathrm{kg}^{-1}$, $26.45 \mathrm{MJ} \mathrm{kg}^{-1}, 26.92 \mathrm{MJ} \mathrm{kg}^{-1}, 29.18 \mathrm{MJ} \mathrm{kg}^{-1}, 29.62 \mathrm{MJ} \mathrm{kg}^{-1}$, $31.07 \mathrm{MJ} \mathrm{kg}{ }^{-1},{ }^{21}$ respectively. Compared with these high performance polymers, the GHC of $p$-POD (19.72 $\mathrm{MJ} \mathrm{kg}^{-1}$ ) is only higher than that of PTFE; moreover, the GHC of POD decreases with the increase in the content of BCPPO. Therefore, it can be concluded that POD fiber has excellent fire resistance, and it can also be further enhanced after introducing the TPO unit.

\section{Morphologies of POD fibers after combustion}

The morphologies of the POD fibers before and after combustion were observed using SEM (Fig. 6). It can be found that POD fibers can keep their original shapes well after combustion, illustrating POD is a kind of an excellent refractory material. The combustion residue of the $p$-POD fiber shows a hollow structure, and many particulate matters deposited and formed its rough surface, suggesting that $p$-POD fibers were decomposed into a larger amount of volatile gases during combustion process. The combustion residue of pho-POD-7.5 fiber shows a porous structure without deposited particles on its surface, while the residue of pho-POD-15 fiber shows a smooth, dense and non-porous carbon layer, which is consistent with the variation of the char yield determined by TGA. The results illustrate that the amount of volatile gases reduces after introducing the TPO units into POD molecular chains, and the dense carbon layer can effectively play a role of oxygen barrier, thereby enhancing the flame resistance. Based on the above discussion, it can be said that the flame resistance mechanism of the phoPOD is responsible for the condensed phase effect.

\section{Volatile gases released out during the thermal degradation}

TG-FTIR was used to identify the gas products during the thermal degradation process of POD fibers. The FT-IR spectra of the volatile gas released out during the thermal degradation of $p$-POD (A) and pho-POD-15 (B) fibers are shown in Fig. 7. It can be see that the bands at about $3700 \mathrm{~cm}^{-1}$ are ascribed to

Table 4 Pyrolysis products from $p$-POD and pho-POD-15

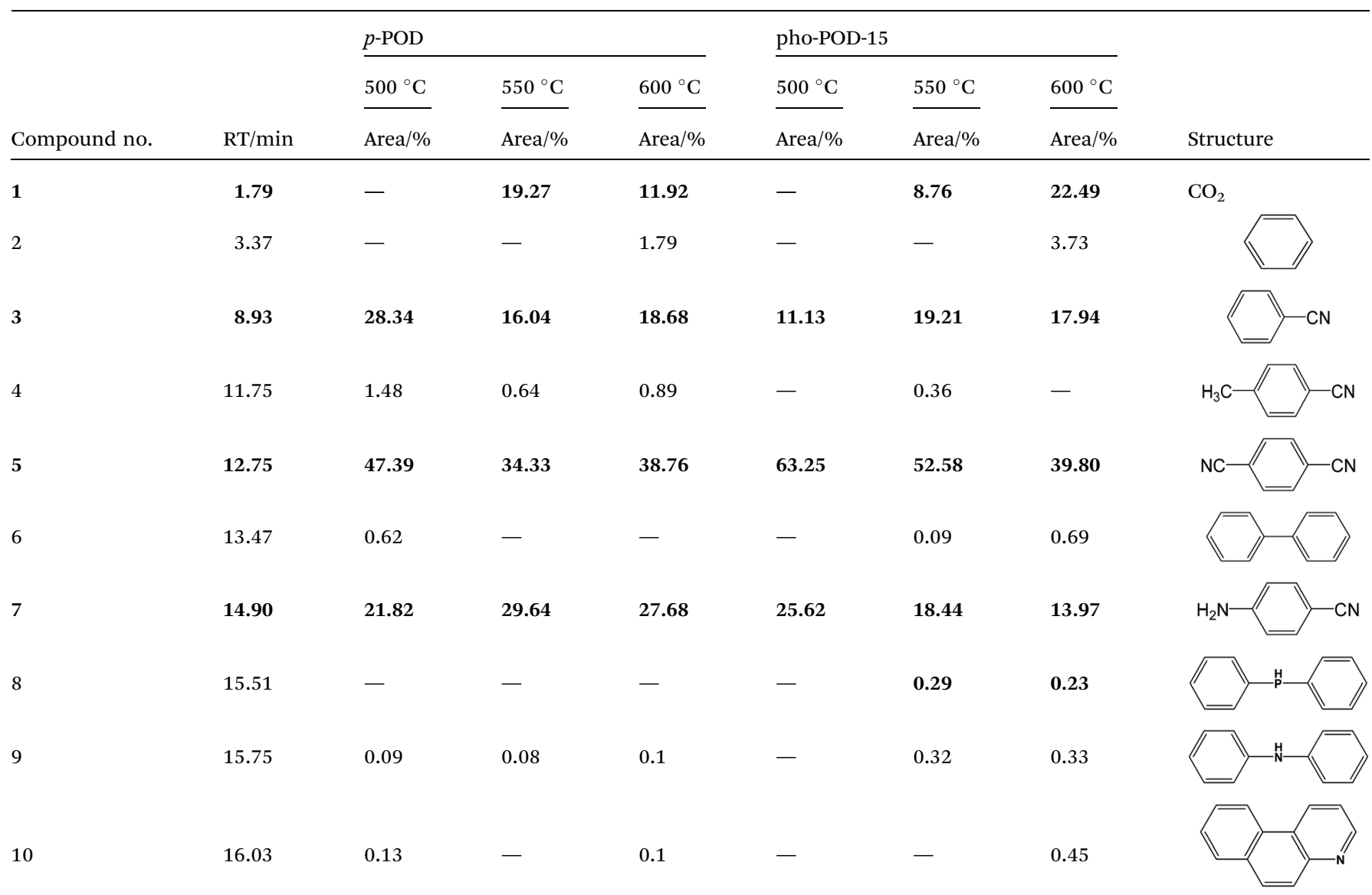


the stretching vibration of $\mathrm{H}_{2} \mathrm{O}$, and the band at about $2300 \mathrm{~cm}^{-1}$ and $1600 \mathrm{~cm}^{-1}$ is attributed to the stretching vibration of $\mathrm{CO}_{2}$. The peak at $1100 \mathrm{~cm}^{-1}$ could be assigned to the stretching vibration of $\mathrm{C}-\mathrm{O}$ bone. The absorptions at $670 \mathrm{~cm}^{-1}$ might be associated with the stretching vibration of HCN. For the volatile gases of pho-POD-15 fiber, FT-IR Spectra also show the stretching vibration of $\mathrm{H}_{2} \mathrm{O}, \mathrm{CO}_{2}, \mathrm{C}-\mathrm{O}$ bone and $\mathrm{HCN}$. Besides new band appears at about $1350 \mathrm{~cm}^{-1}$, which can be ascribed to the stretching vibration of $\mathrm{P}=\mathrm{O}$ and $\mathrm{PO}_{2} /$ $\mathrm{PO}_{3}$ in phosphate-carbon complexes. The presence of polyphosphate can dilute the amount of oxygen; moreover, it can catalyze the formation of char, and this conclusion is consistent with the analysis of TGA. Therefore, it can be inferred that phosphorus can act in the vapor phase by a radical mechanism to interrupt the exothermic processes and to suppress combustion.

\section{Volatile pyrolysis products of POD fibers}

To further understand the effect of introducing TPO units on the thermal stability and flame resistance of the POD fibers, PyGC/MS technique was employed to analyze the volatile products arising from the decomposition of $p$-POD and pho-POD-15 fibers. Using TGA data as a guide, pyrolysis studies were consecutively performed at the initial, maximum and final decomposition temperatures of the proposed polymer. Considering the faster heating rate employed in Py-GC/MS $\left(260{ }^{\circ} \mathrm{C} \min ^{-1}\right.$ ) as compared with the TGA testing $\left(20{ }^{\circ} \mathrm{C} \min ^{-1}\right)$, the pyrolysis experiments were performed at $500{ }^{\circ} \mathrm{C}, 550{ }^{\circ} \mathrm{C}$ and $600{ }^{\circ} \mathrm{C}$, respectively. The volatile pyrolysis products can be separated by a gas chromatography column, and the total ion chromatograms of the pyrolysates of $p$-POD and pho-POD-15 in helium at different temperatures are shown in Fig. 8. It can be seen from Fig. 8, the relative intensities of the pyrolysates of pho-POD-15 are lower than those of $p$-POD at the same pyrolysis temperature, indicating the TPO unit of phoPOD can inhibit the production of the volatile products, which is also consistent with the results obtained from TG analysis and the morphological analysis of the POD fibers after combustion.

The mass spectra of the volatile pyrolysis products of the POD fibers are shown in Fig. S1-S3 in ESI. $\dagger$ In the Table 4, the pyrolysates of the $p$-POD and pho-POD-15 fibers are listed in the order retention time. There are five mainly pyrolysis products for the $p$-POD and pho-POD fibers, including carbon dioxide (compound 1), benzonitrile (compound 3), terephthalonitrile (compound 5) and 4-aminobenzonitrile (compound 7), taking excess 90 percent of all the pyrolysis products. It can be concluded that introducing the TPO units does not essentially affect the composition of pyrolysis products. Compared with $p$ POD, pho-POD-15 just has a new phosphorus-containing pyrolysis product, namely diphenylphosphine (compound 8), which comes from the decomposition of the TPO units. However, the relative proportions of the diphenylphosphine only take 0.29 and 0.23 percent of the total products at the pyrolysis temperature of $550{ }^{\circ} \mathrm{C}$ and $600{ }^{\circ} \mathrm{C}$, respectively, indicating that most phosphorus-containing substances are kept in the condensed phase. It can be further confirmed that the mechanism of enhancing the flame retardancy by introducing TPO units is mainly the flame retardation of the condensed phase.

\section{Conclusion}

In the present study, a series of halogen-free flame retardant and phosphorus-containing aromatic poly(1,3,4-oxdiazoles) fibers were fabricated by introducing triaryl phosphine oxide (TPO) units into the main chains of $p$-POD. Although the mechanical properties and thermal stabilities of the phoPOD fibers slightly decreased after introducing the TPO units, the flame resistance of the POD fiber could be improved significantly. Through comparative analysis, the pho-POD-15 fiber showed excellent comprehensive performance. The mechanism of enhancing the flame retardancy by introducing TPO units is mainly the flame retardation of the condensed phase.

\section{Conflicts of interest}

There are no conflicts to declare.

\section{Acknowledgements}

The authors are grateful to the National Key R\&D Program of China for financial support (Project No. 2016YFB1200602). The authors also gratefully acknowledge Ms Chenxu Tian from the College of Polymer Science and Engineering of Sichuan University for her help in measurement and characterization.

\section{References}

1 X. Yang, M. W. Shi, L. A. Dong, Y. Y. Ma, G. D. Ye and J. J. Xu, Polym. Degrad. Stab., 2010, 95, 2467-2473.

2 E. V. Dovbii, A. T. Kalashnik, O. I. Pankina, N. P. Okromchedlidze and S. P. Papkov, Fibre Chem., 1998, 30, 108-110.

3 W. L. Zhou, X. Yang, E. P. Jia, X. Wang, J. J. Xu and G. D. Ye, Polym. Degrad. Stab., 2013, 98, 691-696.

4 Y. H. Mao, W. L. Zhou and J. J. Xu, J. Appl. Polym. Sci., 2015, 132, 42643.

5 X. Yang, X. Y. Chen, L. Duan and X. Q. Ran, J. Polym. Res., 2018, 25, 141.

6 X. Yang, L. Duan, X. J. Cheng and X. Q. Ran, J. Polym. Res., 2016, 23, 87.

7 Y. H. Mao, Q. S. Song and Y. Guan, J. Text. Inst., 2018, 109, 1173-1185.

8 Y. H. Mao, Q. S. Song and Y. Guan, J. Appl. Polym. Sci., 2017, 134, 44745.

9 Y. H. Mao, X. Zheng and Y. Guan, J. Appl. Polym. Sci., 2017, 134, 44928.

10 X. Q. Ran, S. X. Yi, L. F. Liang and X. Yang, J. Polym. Res., 2014, 21, 597.

11 X. S. Zhang, X. N. Tang, R. Wang, R. Wang, X. Yan and M. W. Shi, Fibers Polym., 2017, 18, 1421-1430. 
12 O. I. Pankina, T. M. Ogneva, N. P. Okromchedlidze and V. D. Manzurov, Fibre Chem., 1984, 15, 411-413.

13 L. S. Wang, X. L. Wang and G. L. Yan, Polym. Degrad. Stab., 2000, 69, 127-130.

14 H. B. Chen, Q. Zhou, X. Dong, Y. Zhang, L. Chen and Y. Z. Wang, Polym. Degrad. Stab., 2012, 97, 905-913.

15 H. B. Chen, Y. Zhang, L. Chen, W. Wang, B. Zhao and Y. Z. Wang, Polym. Adv. Technol., 2012, 23, 1276-1282.

16 C. X. Tian, P. Q. Liu, G. D. Ye and J. J. Xu, J. Appl. Polym. Sci., 2013, 130, 3521-3529.
17 X. F. Yang, Q. L. Li, Z. P. Chen and H. L. Han, Bull. Mater. Sci., 2009, 32, 375-380.

18 X. T. Chen, M. Zhang and X. D. Tang, Chin. J. Polym. Sci., 2008, 26, 793-797.

19 Z. X. Zhang, W. T. Li, G. D. Ye and J. J. Xu, Plast., Rubber Compos., 2007, 36, 343-349.

20 Z. X. Zhang, G. D. Ye, W. T. Li, T. Li and J. J. Xu, J. Appl. Polym. Sci., 2009, 114, 1485-1493.

21 R. N. Walters, S. M. Hackett and R. E. Lyon, Fire Mater., 2000, 24, 245-252. 